\title{
Modal Parameters Directly Estimated from Power Spectral Densities or Correlation Functions in Output-Only Analysis
}

\author{
A. Agneni ${ }^{\dagger}$, L. Balis Crema, and G. Coppotelli \\ Dipartimento di Ingegneria Meccanica e Aerospaziale, Università di Roma "La Sapienza", Roma, Italy
}

Keywords

Structural Dynamics, Experimental Modal

Analysis, Operational Modal Analysis

\section{Correspondence}

G. Coppotelli,

Dipartimento di Ingegneria Meccanica e

Aerospaziale,

Università di Roma "La Sapienza",

Via Eudossiana 18,

00184 Roma,

Italy

Email: giuliano.coppotelli@uniroma1.it

URL: http://www.dima.uniroma1.it/docenti/

g.coppotelli/index.html

${ }^{\dagger}$ Deceased

Luigi Balis Crema and Giuliano Coppotelli

wish to dedicate this "merging" paper to the memory of our friend and colleague

Alessandro Agneni who prematurely passed away on Aug. 16, 2011.

Received: June 15, 2011;

accepted: October 16, 2013

doi:10.1111/ext.12067

\begin{abstract}
Output-only analysis considers the system excited either by operative or by natural forces, in both cases the input loading which excites the structure is considered, at least in a limited frequency band, as a white noise. Because it is not possible to find directly the spectra, it is necessary to pass through the correlation functions so as to apply the Wiener-Khintchine theorem in order to find the power spectral densities (PSDs). In the past, the modal parameters have been essentially derived by approaches that manipulated the functions mentioned above. In this paper, the modal parameters will be directly estimated from the PSDs, in the frequency domain, and from the correlation functions in the time domain. A particular attention is devoted to the problems regarding the damping ratio overestimation. This effect due to the limited time window in the correlation function estimate is highly present in the low-frequency modes and could bring to large estimation errors. Experimental examples, carried out both on cantilever beams and on an helicopter blade, are presented. They show the problems related to the techniques using the PSDs, or the correlation functions and the possibility to overcome the overestimation of the damping factors due to the triangular window, also known as Bartlett window.
\end{abstract}

\section{Introduction}

Several experimental investigations rely on the operational modal analysis due to the powerful and versatile developed algorithms. Among all the vibration-based applications that currently benefit of such approach-structural updating, damage assessment, fault detection, vibration and noise control, ${ }^{1,2}$ - are the most remarkable. All the operational modal analysis techniques are based on the assumption that multiple non-deterministic inputs result to be a broadband Gaussian random excitation loading, whose spectrum can be considered constant almost in the frequency range of interest. Due to the non-deterministic input, the use of correlation functions is necessary to get the power spectral densities (PSDs). On the basis of the above said hypothesis, the developed methods demonstrated that it is possible to estimate the modal parameters of a structure, that is, natural frequencies, damping ratios, and mode shapes, from the knowledge of the PSDs or the correlation functions. ${ }^{2-7}$ Moreover, time-domainbased techniques are proposed in Refs. 8 and 9 where a state-space formulation is solved in time domain by an orthogonal-projection technique. In this paper, two direct approaches to estimate the modal parameters are proposed. The first one provides the modal parameters from the PSDs by using the least-square polynomial ratio technique. The other one supplies the same information directly from the correlation functions by the exponential least-square fitting (Prony approach). A common practice to eliminate the residual noise from the measurements consists on dividing the data contained in the recording window and averaging the correlation functions derived from the partial data blocks. However, the correlations (performed on limited recorded time functions) 
result to be biased by the triangular function, usually called Bartlett window. Its influence is different in the time domain or in the frequency domain. In the first case, the bias generally has a greater effect on the lowest frequencies present in the base frequency band of interest. For the highest frequency modes, which decay faster than the biasing triangular function, the damping ratios could be estimated with acceptable errors (with the same acquisition parameters). For the mid-frequency modes, the triangular function substantially affects the terminal part of the time signal, therefore it seems to be possible to obtain the damping ratios, with sufficient accuracy, by using the initial part of the decay function, instead of the total signal. On the contrary, as regarding the frequency domain approaches, a complete biased frequency response function (FRF), matrix can be derived by the PSD matrix of the output responses, in fact all the data are used in order to gain the FRF. The experimental tests, carried out on cantilever beams and on an helicopter blade, showed the troubles mentioned above. Some suggestions (also derived from the experimental tests) to overcome the problems linked to damping estimates are offered.

\section{Theoretical Background}

Assuming the responses of a linear system represented by stationary random functions, the autocorrelation function $\Phi$ can be considered as the expected value of the product of $f(t)$ (called $f$ for the sake of simplicity) times the value assumed by the function in the time instant $(t+\tau)\left(f_{\tau}\right),{ }^{10}$ :

$$
\Phi_{f f}(\tau)=E\left\{f, f_{\tau}\right\}=\int_{-\infty}^{+\infty} \int_{-\infty}^{+\infty} f f_{\tau} p_{\tau}\left(f, f_{\tau}\right) \mathrm{d} f \mathrm{~d} f_{\tau}
$$

where $p_{\tau}\left(f, f_{\tau}\right)$ is the joint probability density function (joint pdf). Because the pdf is normally unknown, supposing the ergodicity of the random functions, it is possible to apply the relationship that allows one to get the correlation for power signals, that is:

$$
\Phi_{f f}(\tau)=\lim _{T \rightarrow \infty}\left(\frac{1}{T}\right) \int_{-T / 2}^{+T / 2} f(t) f(t+\tau) \mathrm{d} t
$$

and obviously:

$$
\Phi_{f g}(\tau)=\lim _{T \rightarrow \infty}\left(\frac{1}{T}\right) \int_{-T / 2}^{+T / 2} f(t) g(t+\tau) \mathrm{d} t
$$

for the cross-correlation function between the functions $f(t)$ and $g(t)$. Because we have sampled signals, instead of the previous relations, the estimates must be performed as the mean lagged product:

$$
R_{f g}(m)=\left(\frac{1}{N}\right) \sum_{n=0}^{N-m-1} f_{n} g_{n+m}
$$

where $N$ is the number of samples [equal for the two sequences $f(t)$ and $g(t)]$ and $R_{f g}(m)$ represents an estimation of the linear correlation between the two signals. As one can see, from Eq. $4, R_{f g}(m)$ [or $R_{f f}(m)$ ] has $2 N-1$ sample points, but it is possible to use only one half of the correlation function in order to get the modal parameters, that is, natural frequencies, damping factors, and mode shapes. The autocorrelation function is an even function, which has a real symmetrical spectrum, but if we only get the part of the correlation functions valid for positive times, as shown in Ref. 7, it is possible, also in this case, to use commercial codes (which generally use polynomial ratio least-square fitting of complex functions) in order to estimate the modal parameters in the frequency domain. So, as mentioned above, in the case of autocorrelation functions it is possible to consider only the causal part of the signal by taking account of the original even function-denoted by the superscript e (divided by 2) — and its relative odd part (superscript o):

$$
\Phi_{x_{\mathrm{i}} x_{\mathrm{i}}}^{\mathrm{o}}(\tau)=\Phi_{x_{\mathrm{i}} x_{\mathrm{i}}}^{\mathrm{e}}(\tau) \cdot \operatorname{sgn}(\tau)
$$

where $\operatorname{sgn}(\cdot)$ represents the sign function. The previous relationship, applying the Fourier transform to both sides, provides:

$$
G_{x_{i} x_{i}}^{\mathrm{o}}(f)=G_{x_{i} x_{i}}^{\mathrm{e}}(f) *\left(-j \frac{1}{\pi f}\right)
$$

where $f$ stands for frequency, measured in Hertz. The convolution, indicated for the sake of simplicity with the asterisk, is the Hilbert transform, denoted as superscript ^, of $G_{x_{\mathrm{i}} x_{\mathrm{i}}}^{\mathrm{e}}(f)$, so the PSD of the causal part of the autocorrelation function, for $\tau>0$, is given by:

$$
G_{x_{\mathrm{i}} x_{\mathrm{i}}}(f)=G_{x_{i} x_{i}}^{\mathrm{e}}(f)-j \widehat{G}_{x_{i} x_{i}}^{\mathrm{e}}(f)
$$

When different locations of measurements are considered, for instance the $x_{i}(t)$ and the $x_{j}(t)$ points, the cross-correlation function, $\Phi_{x_{i} x_{j}}(\tau)$, is derived (of course this function is generally neither an odd or an even function). It is possible to split the cross correlation so as to get the even part, ${ }^{7}$ :

$$
\Phi_{x_{i} x_{j}}^{\mathrm{e}}(\tau)=\frac{\Phi_{x_{i} x_{j}}(\tau)+\Phi_{x_{i} x_{j}}(-\tau)}{2}
$$

and the odd part:

$$
\Phi_{x_{i} x_{j}}^{\mathrm{o}}(\tau)=\frac{\Phi_{x_{i} x_{j}}(\tau)-\Phi_{x_{i} x_{j}}(-\tau)}{2}
$$


Now summing up the two relations, one can obtain the part of the signal valid for $\tau \geq 0$ :

$$
\Phi_{x_{i} x_{j}}(\tau)=\Phi_{x_{i} x_{j}}^{\mathrm{e}}(\tau)+\Phi_{x_{i} x_{j}}^{\mathrm{o}}(\tau)
$$

The previous equation defines a causal signal, which can be written as in Eq. 5:

$$
\Phi_{x_{i} x_{j}}(\tau)=\Phi_{x_{i} x_{j}}^{\mathrm{e}}(\tau)+\Phi_{x_{i} x_{j}}^{\mathrm{e}}(\tau) \cdot \operatorname{sgn}(\tau)
$$

By performing the Fourier transform, it is possible to derive the following relationship:

$$
G_{x_{i} x_{j}}(f)=G_{x_{i} x_{j}}^{\mathrm{e}}(f)-j \widehat{G}_{x_{i} x_{j}}^{\mathrm{e}}(f)
$$

which is completely similar to the one obtained in Eq. 7. In this way, taking account of the part of the correlation function (both auto and cross) valid for time greater than zero, it is always possible to get the spectral densities, with their real and imaginary parts different from zero that can be fitted with the commercial codes as well. Actually, the correlation function, numerically derived, results to be biased by the "Bartlett" (triangular) window, $w(m)$ as reported in Ref. 13, which is the autocorrelation function of the rectangular window which, in turn, determines the finite length of the recorded data. In the previous reference, it was demonstrated that for a given sampling point index $m$, the triangular window is given by:

$$
w(m)=\left[1-\frac{m}{N}\right]
$$

Because for $N \rightarrow \infty$ the value of $w(m)$ tends to the unity, the correlation function estimate results asymptotically unbiased. ${ }^{11,12}$ So, some troubles might derive from the presence of the above-mentioned triangular window in a limited recording data. In fact, the PSDs also take account of it, and since the Fourier transform consider all the time recorded data, the bias on the damping factor estimates can be relevant. On the contrary, performing the evaluation in the time domain, this effect could be minimized. Following Ref. 13, if the exponential decreasing function of a mode intersects the triangular window at the time $\tau_{\lim }$, that is if:

$$
\frac{\tau_{\lim }}{\tau_{\mathrm{C}}}=-\ln \left[1-\frac{\tau_{\lim }}{T}\right]
$$

(where $\tau_{c}$ is the time constant of the considered mode) it is possible to derive that the limit time ought to be in the order of $[1 / 10] \tau_{c}$, supposing that the time constant is much smaller than the recording time $T$. Therefore, if the part of the time signal which satisfies the previous constraint is considered, for the damping estimation (in the time domain), a smaller bias can be obtained. This criteria is reported in Fig. 1 where

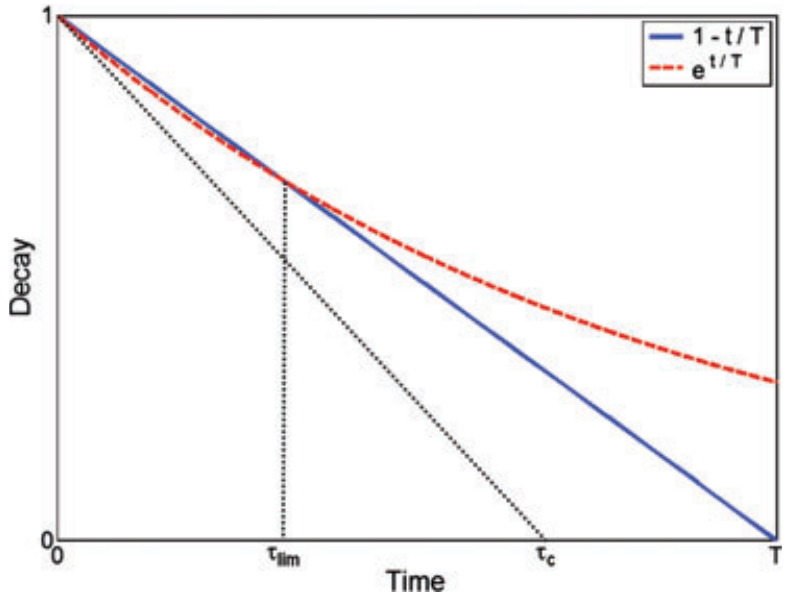

Figure 1 Schematic representation of the effects of the Bartlett (triangular) window.

both the triangular window and the decay of the correlation function are plotted as function of time as well as the characteristic times $\tau_{\lim }$ and $\tau_{\mathrm{c}}$. As one can see, the lower is the value of $\tau_{\lim }$ (with respect to the value of $\tau_{c}$ ) the lower is the bias induced by the triangular window (represented by the solid blue line). Otherwise, when the damping is so low that the correlation function has a triangular behavior, much longer time sequences must be considered before performing the correlations. Obviously, a trade-off between the length of the time sequences and the bias due to the triangular window must be considered. In fact, shorter time sequences allow one to eliminate more residual noise but, on the other hand, they present a greater bias due to the triangular window.

\section{Experimental Tests}

\section{Cantilever beams}

The procedures, presented in this paper, have been applied to identify the modal parameters-natural frequencies, damping ratios, and mode shapes-from the data gathered from a cantilever aluminum beam, whose dimensions were $0.2 \times 0.0154 \times 0.00285 \mathrm{~m}$. The dynamic excitation was provided by a pencil crawling on one side of the beam, whereas the output random response acceleration was measured by four accelerometers, equally distributed along the beam span. The sampling points contained in the time window were 16,384 for a recording time equal to 20.48 s. In Fig. 2, the time responses recorded by the four accelerometers are reported. The (classical) time-domain-based Prony approach was used to estimate the modal parameters in the time domain. This 


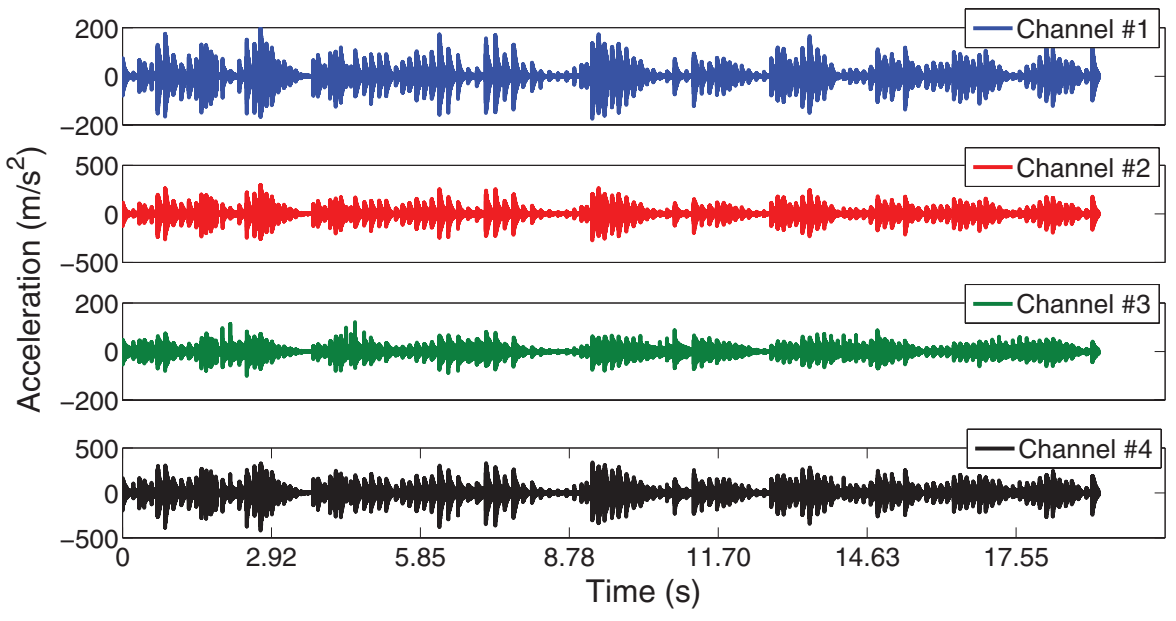

Figure 2 Recorded acceleration response for the cantilever beam excited by a crawling pencil. method performs a least-square estimate of the modal parameters by using exponential functions to fit the positive part, $\tau \geq 0$, of the correlation functions. In order to minimize the presence of residual random noise in such correlation functions, the recorded data was averaged over 32 time-data blocks, obtained by dividing the whole recorded data length into 32 time segments. The estimating process considered only the initial sampling points $(1-151)$ and an order of the modal model equal to 10 , that is 10 modes/poles were used to curve fitting the response data. These estimates were denoted with the superscript $t$. The sum of the correlations functions was also transformed into the frequency domain and the evaluation of the modal parameters was carried out with a multi-modal least-square fitting performed by a polynomial ratio (the estimates have been indicated with the superscript $f$ ). Also for these estimates, the size of the model was 10 as in the previous case. It is worthwhile noting that because the modes are well separated in the frequency domain, the chosen model order is high enough to guarantee an acceptable level of accuracy. The poles identified using a model size equal to 10 differ less than $1 \%$ (in both the real and imaginary part) with respect to the corresponding poles estimated using a model size equal to 9 or even lower. The mean values - over the four outputs - of the first two natural frequencies are presented in Table 1. The same procedure has been adopted for the damping ratio measurements, whose mean values have been shown in Table 2. In the same tables, the natural frequencies and damping ratios estimated using the more traditional input-output-based estimating technique are also reported, as reference. For this case, a modal hammer was used to excite the structure. These last modal parameters are denoted with the superscript i-o. As a general comment, both the natural
Table 1 Mean values of the natural frequency estimates for the cantilever beam excited by a crawling pencil

\begin{tabular}{lrrr}
\hline Mode \# & $f_{n}^{t}(\mathrm{~Hz})$ & $f_{n}^{f}(\mathrm{~Hz})$ & $f_{n}^{i-o}(\mathrm{~Hz})$ \\
\hline 1 & 51.11 & 51.14 & 50.98 \\
2 & 319.21 & 318.92 & 318.07 \\
\hline
\end{tabular}

Table 2 Mean values of the damping ratio estimates for the cantilever beam excited by a crawling pencil

\begin{tabular}{lccc}
\hline Mode \# & $\zeta_{n}^{t}(\%)$ & $\zeta_{n}^{f}(\%)$ & $\zeta_{n}^{i-o}(\%)$ \\
\hline 1 & 1.09 & 1.31 & 0.56 \\
2 & 0.50 & 0.46 & 0.40 \\
\hline
\end{tabular}

frequencies and the damping ratios estimated using output-only data are consistent with those gained using the input-output approach, also considering higher order modes (not reported for the sake of brevity). Specifically, the natural frequencies are practically the same, whereas the damping ratios present a non-negligible error for the lowest natural frequency, and that is due to the effect of the triangular (Bartlett) window. Indeed, the exponential function at the last data point, considered in the time-domainbased Prony estimation, had the value 0.5, against 0.7 of the Bartlett window, thus with a very limited biasing effect. On the contrary, in the frequency domain, the full window contributes to the damping bias resulting then in lower accuracy. However, the comparison of the corresponding mode shapes, estimated by the correlation functions or power spectral densities, is excellent, as depicted in Fig. 3, where the first and the second mode, estimated using the proposed approach in the time domain, are compared with those achieved in the frequency domain. 


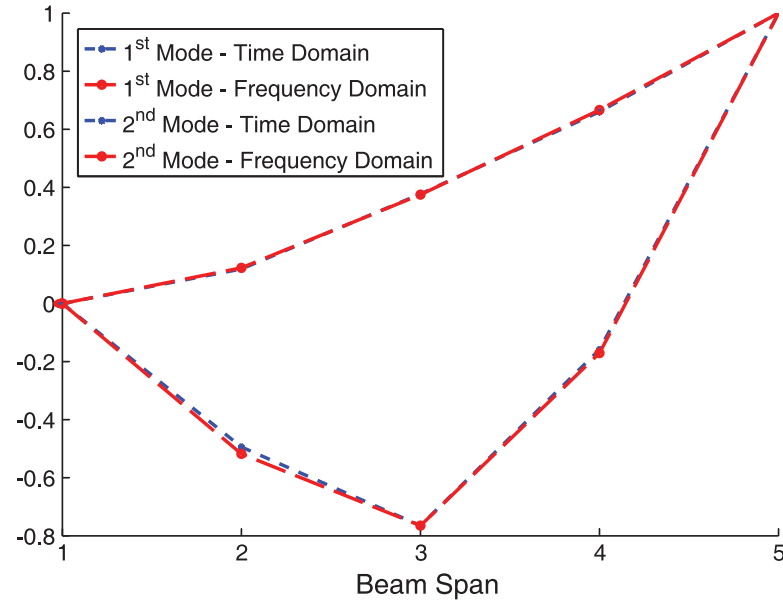

Figure 3 Mode shape estimate for the cantilever beam excited by a crawling pencil.

Another test has been carried out on an aluminum cantilever beam (dimensions: $0.16 \times$ $0.015 \times 0.00295 \mathrm{~m})$, excited by a loudspeaker positioned at $0.08 \mathrm{~m}$ from the second node (starting from the clamped end) and driven with a broadband noise, see Fig. 4 for the graphical representation of the recorded time histories. The frequency band of analysis was set equal to $0-2048 \mathrm{~Hz}$ and the sampling points were 16,384 for a total recording time of $T=4 \mathrm{~s}$. Actually the random noise had a spectrum which excited the first bending mode so badly that it was practically impossible to identify it neither in the frequency nor in the time domain. Also in this case, the natural frequencies and the mode shapes are almost the same (Table 3 and Fig. 5). On the
Table 3 Mean values of the natural frequency estimates for the cantilever beam excited by a loudspeaker

\begin{tabular}{lrr}
\hline Mode \# & $f_{n}^{t}(\mathrm{~Hz})$ & $f_{n}^{f}(\mathrm{~Hz})$ \\
\hline 2 & 508.23 & 509.26 \\
3 & 1411.52 & 1411.05 \\
\hline
\end{tabular}

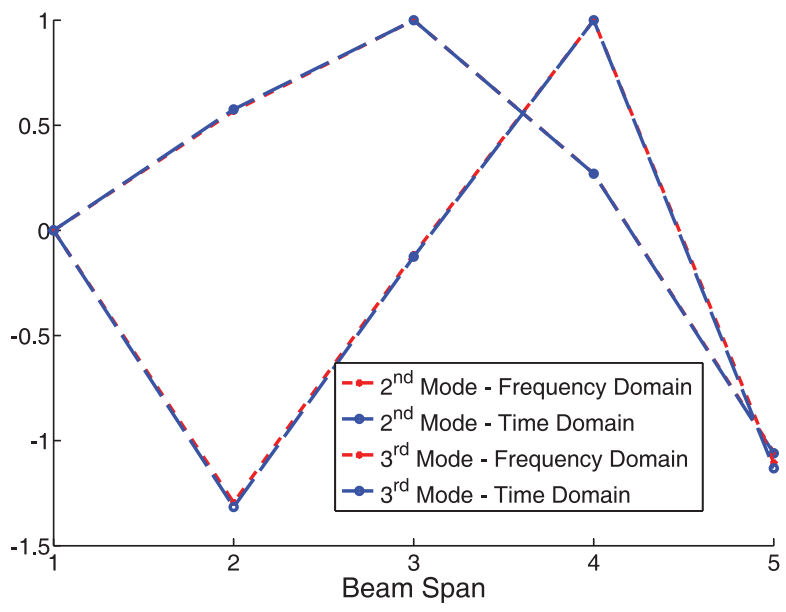

Figure 5 Mode shape estimate for the cantilever beam excited by a loudspeaker.

contrary, about $20 \%$ relative error-between the two procedures - was found for the damping ratios associated with the first identified mode (mode \# 2), as reported in Table 4. For this mode, although the time constant $\tau_{\mathrm{c}}$ is equal to about $0.03(\mathrm{~s})=T$, the value of the decay function, evaluated at the 151 st sampling point, is 0.3 , see Eq. 13, whereas the triangular function $w(m)$ is equal to 0.7 . If $m=50$

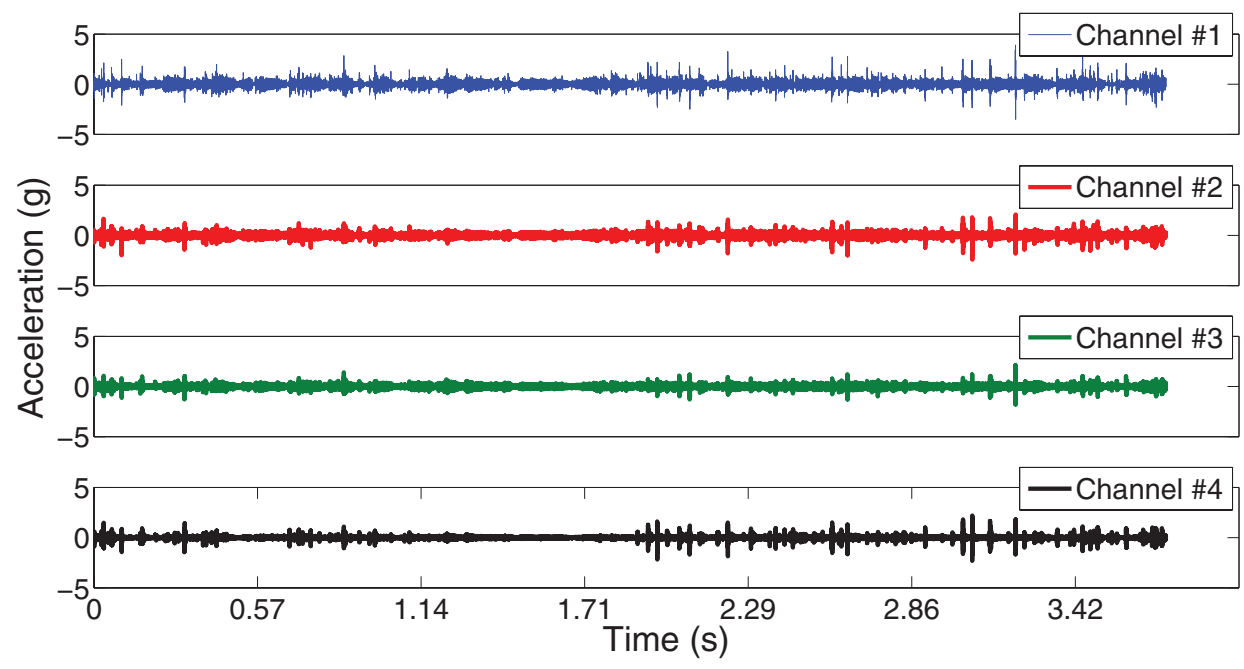

Figure 4 Recorded acceleration response for the cantilever beam excited by a loudspeaker. 
Table 4 Mean values of the damping ratio estimates for the cantilever beam excited by a loudspeaker

\begin{tabular}{lcc}
\hline Mode \# & $\zeta_{n}^{t}(\%)$ & $\zeta_{n}^{f}(\%)$ \\
\hline 2 & 0.94 & 1.14 \\
3 & 0.35 & 0.37 \\
\hline
\end{tabular}

were considered in the time estimates, the damping ratio would present a small decrease $\zeta_{1}^{t}=0.87 \%$, whereas the one associated to the second mode would be practically the same $\zeta_{2}^{t}=0.32 \%$ having the same time constant. On the contrary, if $m=200$ were adopted, the presence of the triangular window would have an influence especially on the first identified mode, in fact $\zeta_{1}^{t}=1.02 \%$ and $\zeta_{2}^{t}=0.31 \%$.

\section{AB-204 helicopter blade}

Finally, a third example was carried out to investigate the accuracy of the proposed operational methods when dealing with a real structure. The considered structure was an AB-204 helicopter blade, whose dimensions were: span $6.100 \mathrm{~m}$, chord $0.530 \mathrm{~m}$, and weight $88.0 \mathrm{~kg} .6,14$ The skin, the sandwich core, and the main spar are made by aluminum alloys. The measured frequency band was $0-100 \mathrm{~Hz}$, whereas the sampling points were $2^{15}$. The structure was hung outdoor with rubber bands and therefore it was excited by natural events (wind). The output responses were recorded at 11 spanwise locations. In each location, the acceleration of the leading edge, the $1 / 4$ chord, and the trailing edge were recorded by using three accelerometers. In Fig. 6, the acceleration responses corresponding to the blade section located
Table 5 Natural frequency and damping ratio estimates for the AB-204 helicopter blade from LMS-PolyMax input-output based approach

\begin{tabular}{lcc}
\hline Mode \# & $f_{n}^{i-o}(\mathrm{~Hz})$ & $\zeta_{n}^{i-o}(\%)$ \\
\hline 1 & 7.49 & 0.17 \\
2 & 21.67 & 0.14 \\
3 & 43.70 & 0.39 \\
4 & 44.98 & 0.14 \\
5 & 72.69 & 0.20 \\
\hline
\end{tabular}

at $3 / 4$ of the span are reported. Owing to the large dimensions and the high number of measuring points (33), a roving techniques was employed, so-considering a reference point necessary to get the actual mode shapes -43 time responses had to be recorded using four roving accelerometers. In Table 5, the identified natural frequencies and damping ratios evaluated using the LMS-PolyMax input-outputbased approach are reported as reference. For this modal survey, an electrodynamic shaker was used to provide a white noise excitation, in the analysis frequency band. As in the test cases reported in the previous section, both the time and the frequency domain-based estimating techniques allowed the estimate of a modal model fully compatible with the one achieved using input-output technique. In order to investigate the sensitivity of the proposed techniques to the limited length of the recorded data, the worst cases are here highlighted, that is only the lower and higher natural frequency modes are considered in the following for brevity. Specifically, in Tables 6 and 7 the mean values of natural frequencies and damping ratios of two modes (the first and the fifth ones), obtained by the methods

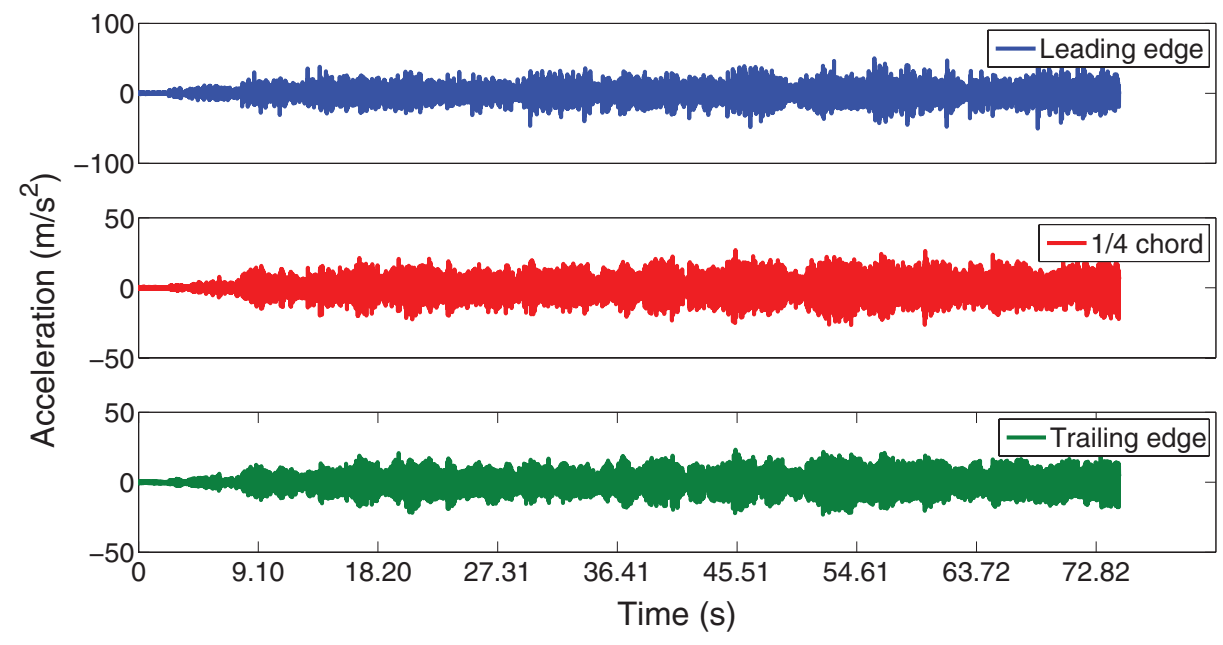

Figure 6 Acceleration response recorded at 3/4 of the span of the AB-204 helicopter blade. 
Table 6 Mean values of the natural frequency estimates for the AB-204 helicopter blade (first and fifth modes over 16 sequences)

\begin{tabular}{lrr}
\hline Mode \# & $f_{n}^{t}(\mathrm{~Hz})$ & $f_{n}^{f}(\mathrm{~Hz})$ \\
\hline 1 & 7.47 & 7.48 \\
5 & 72.63 & 72.63 \\
\hline
\end{tabular}

Table 7 Damping ratio estimates for the AB-204 helicopter blade (first and fifth modes over 16 sequences)

\begin{tabular}{lcc}
\hline Mode \# & $\zeta_{n}^{t}(\%)$ & $\zeta_{n}^{f}(\%)$ \\
\hline 1 & 0.71 & 1.11 \\
5 & 0.15 & 0.13 \\
\hline
\end{tabular}

considered before, are reported. It is worth noting that the identification, over 16 averaged time sequences, was carried out choosing the initial sampling points (1-200) of the time correlation functions, so as to follow the rule of thumb derived by Eq. 14. The number of the complex poles, used in the Prony technique, has been increased up to 30 so as to have a good agreement - at the reference point-between the actual spectrum of the first recorded function and the one synthesized with the estimates derived in the time domain, besides a minimum of the square mean error between the two spectra. That is mainly due to the request of fitting the first bending mode that has been found close to the rigid mode. Afterwards, these estimating parameters were considered frozen and used for all the correlation without any further check. Obviously, due to the considerations made before, if 32 time averages were considered-that is shorter time sequences for the correlation functions-a greater bias should be found, especially for the lower natural frequency mode, see Tables 8 and 9. As happened in the previous examples, the natural frequency estimates were completely similar either by identifying them in the time or in the frequency domain both with a higher and with a lower number of sequences of the original recording on which the correlations have to be performed. The maximum error in the estimate of the natural frequencies, with respect to those from the input-output approach, is lower than $0.3 \%$. Instead, a sensitivity of the damping

Table 8 Mean values of the natural frequency estimates for the AB-204 helicopter blade (first and fifth modes over 32 sequences)

\begin{tabular}{lrr}
\hline Mode \# & $f_{n}^{t}(\mathrm{~Hz})$ & $f_{n}^{f}(\mathrm{~Hz})$ \\
\hline 1 & 7.45 & 7.46 \\
5 & 72.60 & 72.59 \\
\hline
\end{tabular}

Table 9 Damping ratio estimates for the AB-204 helicopter blade (first and fifth modes over 32 sequences)

\begin{tabular}{lcc}
\hline Mode \# & $\zeta_{n}^{t}(\%)$ & $\zeta_{n}^{f}(\%)$ \\
\hline 1 & 1.58 & 1.76 \\
5 & 0.19 & 0.25 \\
\hline
\end{tabular}

ratios to the length of the time sequences is reported. From Tables 7 and 9, it seems that using shorter time sequences, that is the ones obtained with 32 time-data blocks, provide higher damping ratios with respect of using the longer ones (16 time-data block sequences). Also, a sensitivity of the damping ratios to the estimation technique is highlighted. Specifically, a higher damping ratio estimate, associated to the first mode, is obtained when using the PSDs, or the frequency-domain-based technique, with respect to the same estimate, but performed directly using the correlation functions, or the time-domain-based technique. Such values are completely overestimated when comparing them with those gained using the LMS-PolyMax input-output approach. Indeed, the first mode presents a heavy bias due to the triangular window linked to the correlation, also in the case of long time sequences (16 data-block sequences). For these longer time sequences, the triangular window, evaluated at the 200th sampling point, is equal to 0.9 , whereas the exponential function (calculated with the estimated conservative value of the time constant) is 0.7 and 0.5 for the first and the fifth mode, respectively. Therefore, the reduction in the accuracy of the damping ratio associated with that higher order mode was not met due to its greater decay rate. By comparing the damping ratio estimates of such higher order mode with the one from the input-output technique, an acceptable maximum error of about $35 \%$ is evaluated when considering the frequency domain technique. On the other hand, when considering shorter time sequences, 32 time-data blocks, the window had a value of 0.8 against 0.7 for the exponential functions. It is noteworthy that also for the damping estimation in the time domain, for the first mode, there is a contribution of the triangular window, when short sequences are considered. Finally, the mode shapes identified using the direct approach in the time domain are depicted in Figs. 7-11. Such modes are smoother and clearer, also for the two coupled modes (the third and the fourth) than the ones gained from the PSDs. Nevertheless, the mode shapes identified by both the considered techniques are in excellent agreement with those estimated using the input-output-based LMS-PolyMax approach. Indeed, 


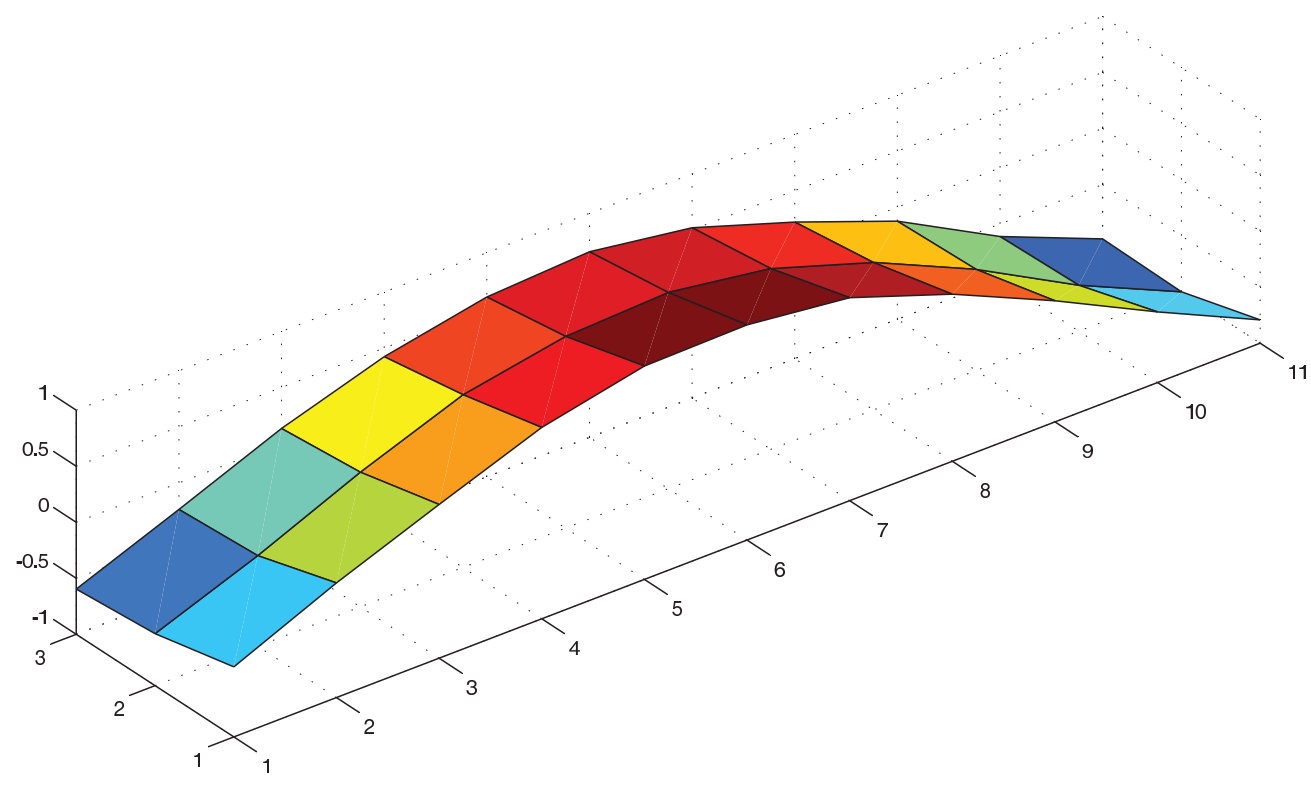

Figure 7 First mode shape estimate of AB-204 helicopter blade from the correlation functions.

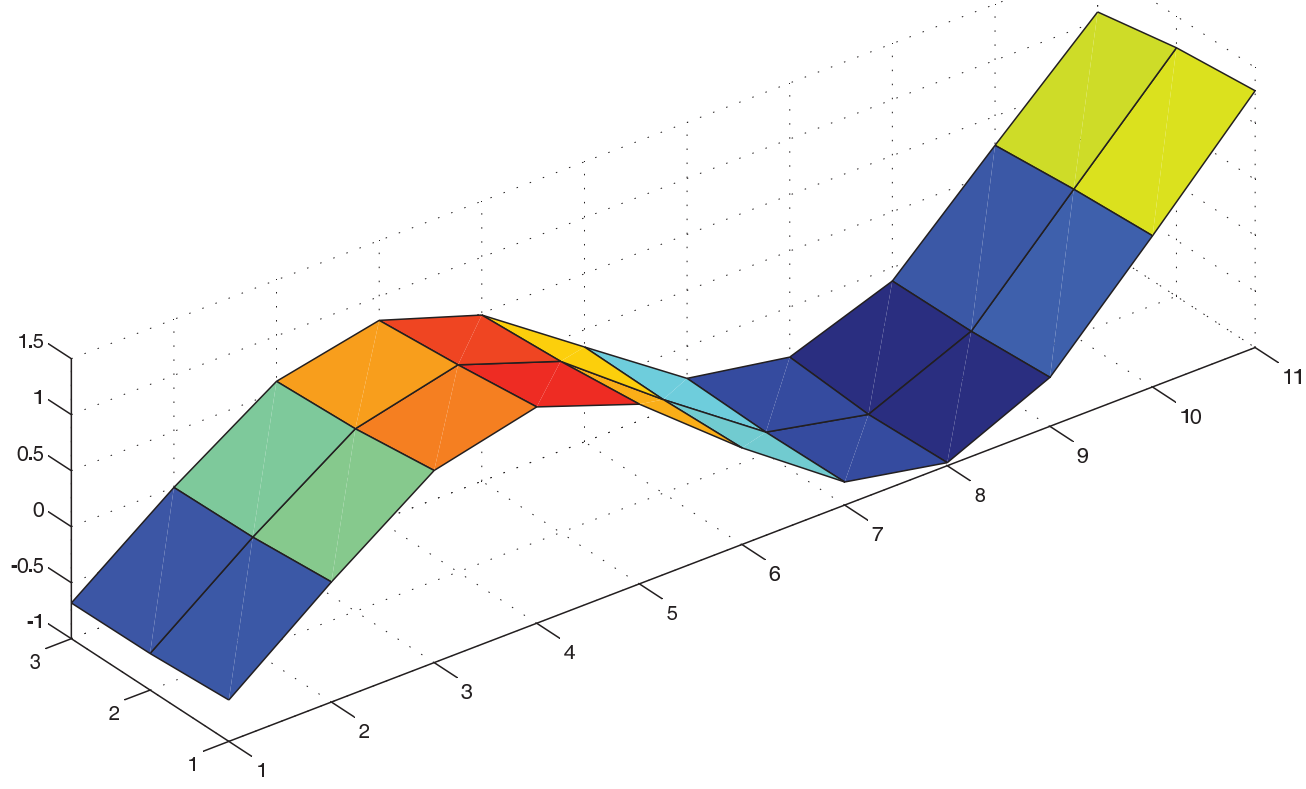

Figure 8 Second mode shape estimate of AB-204 helicopter blade from the correlation functions.

a mode shape correlation up to more than $95 \%$ (in average) has been found. For the sake of shortness, only three modes-achieved from the PSDs-are shown hereafter, and precisely the first, the second, and the fourth mode, Figs. 12-14. It is worth noting that the fourth mode has a frequency very close to the third one and results to be a combination of the out-of-plane bending and torsional modes.

\section{Concluding Remarks}

The examples, shown in this paper, point out the possibility to directly obtain the modal parameters-natural frequencies, damping ratios, and mode shapes - for systems working under their actual operative conditions either from the PSDs or from the autocorrelation functions. Obviously, the assumption of a constant force spectrum-in the 


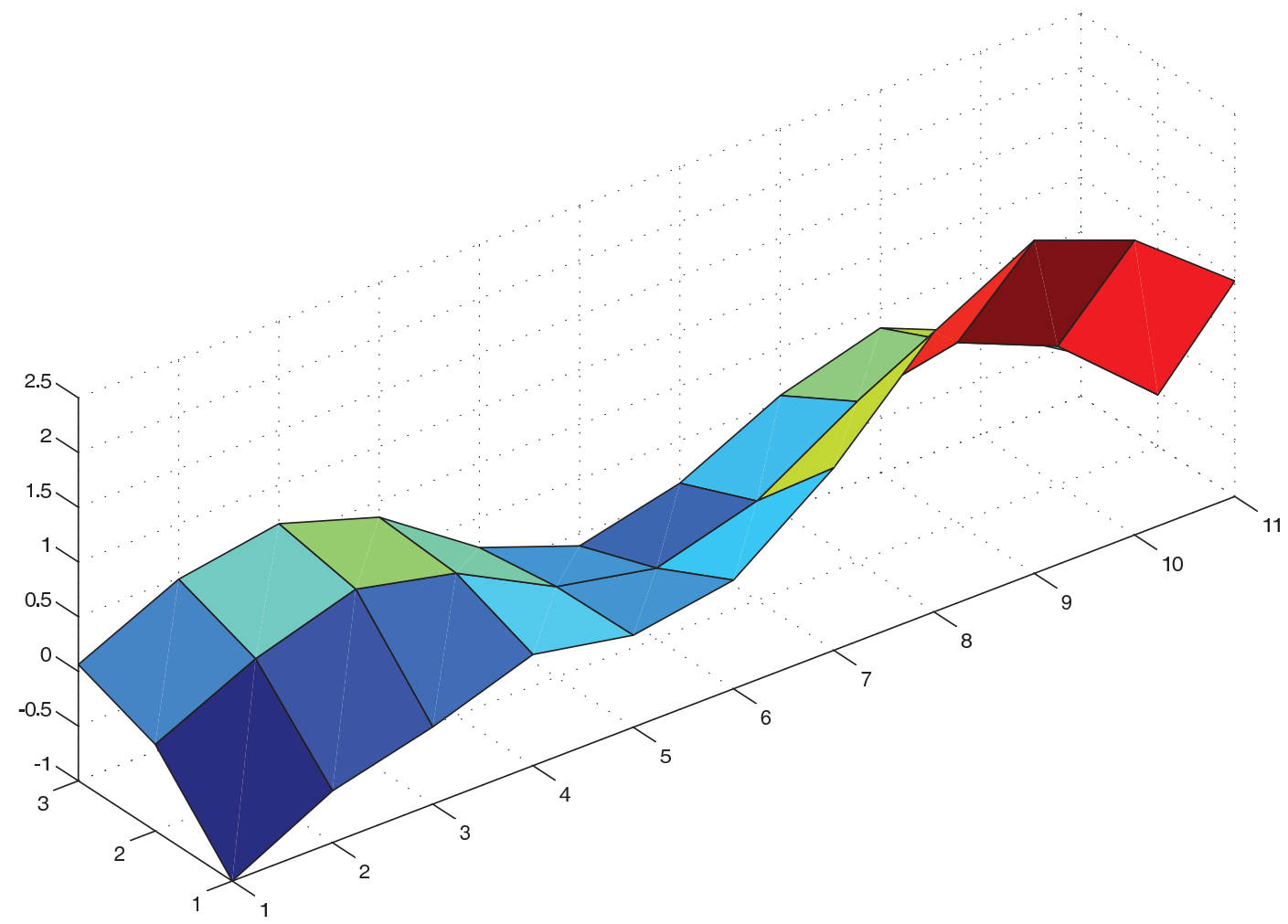

Figure 9 Third mode shape estimate of AB-204 helicopter blade from the correlation functions.

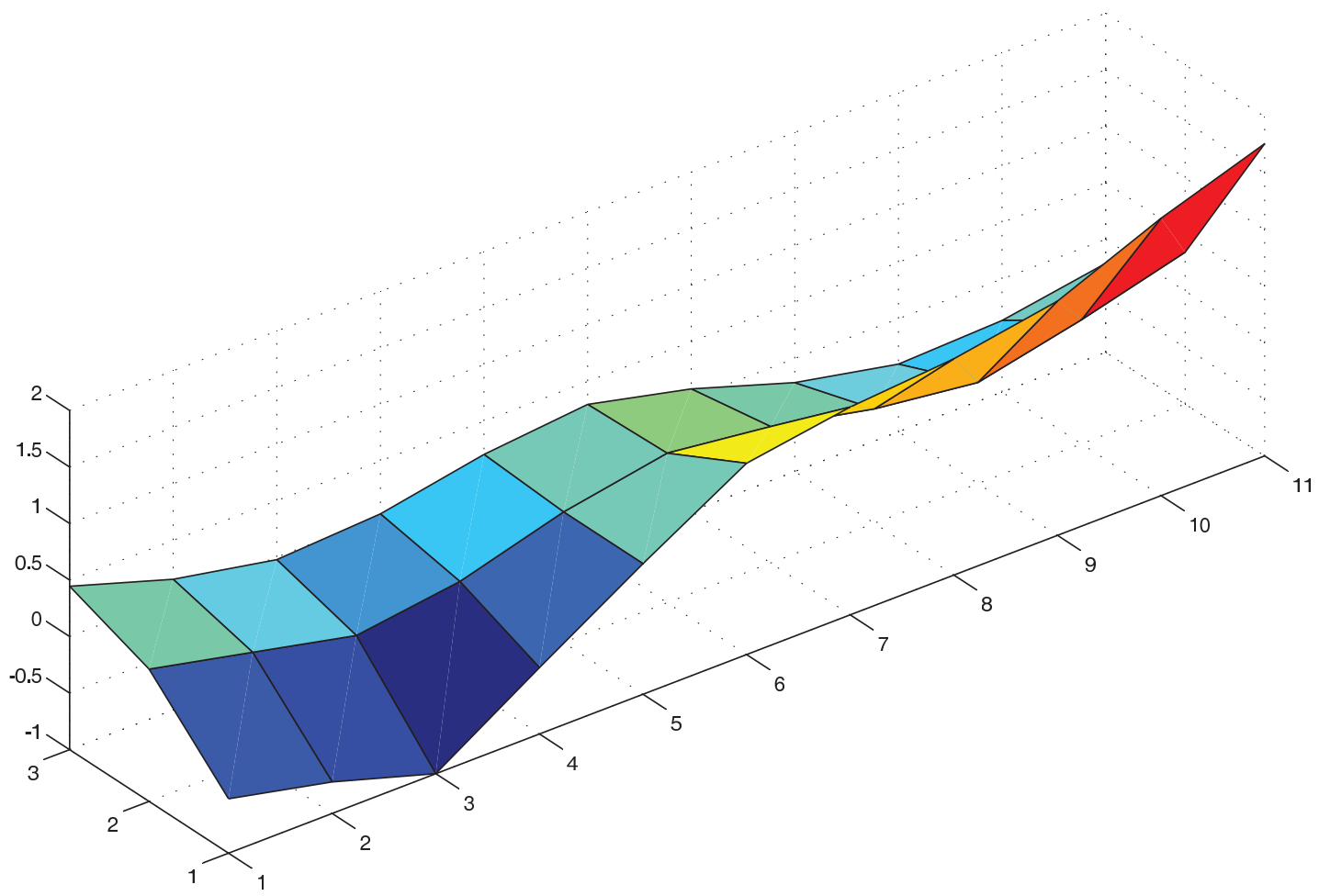

Figure 10 Fourth mode shape estimate of AB-204 helicopter blade from the correlation functions. 


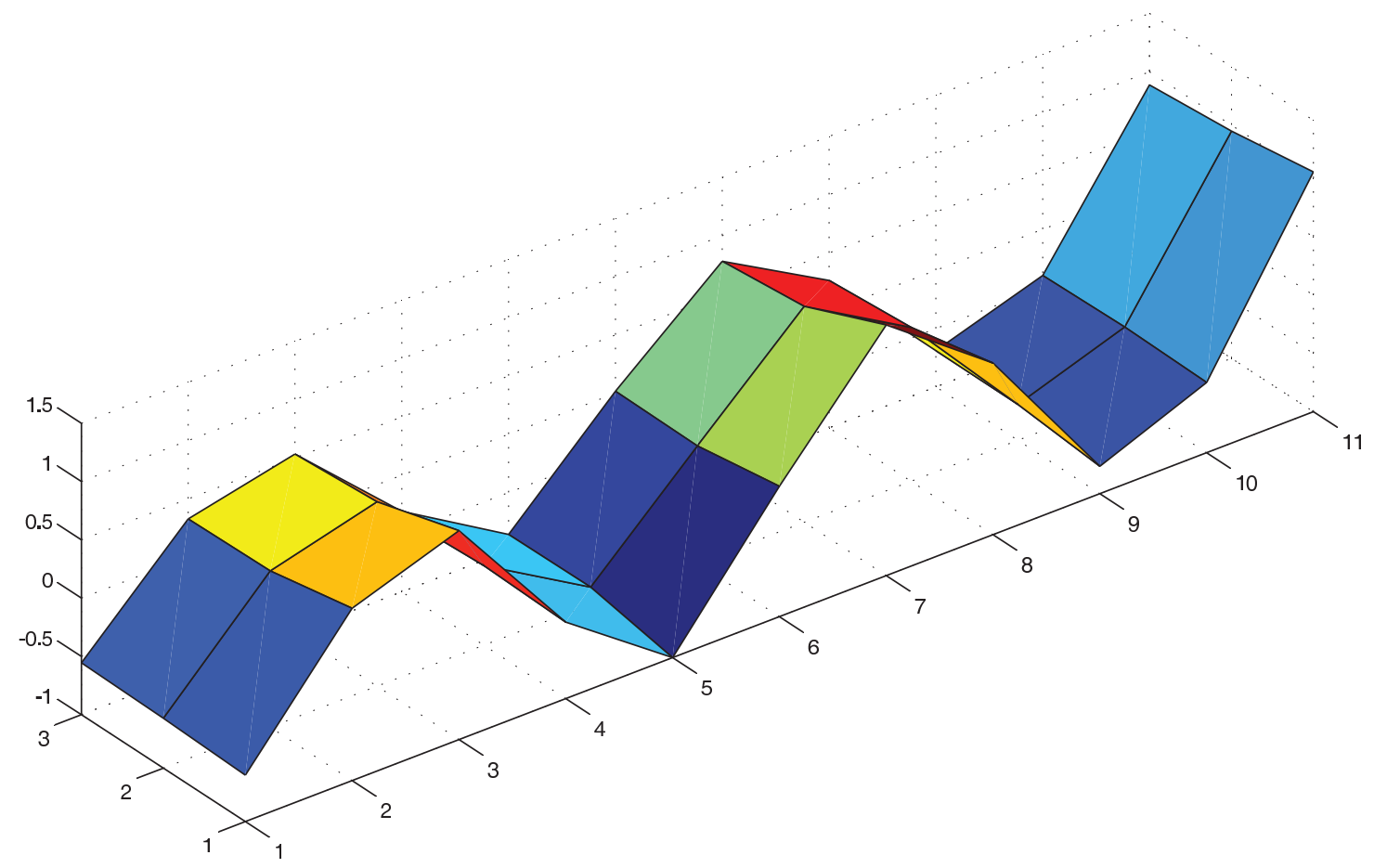

Figure 11 Fifth mode shape estimate of AB-204 helicopter blade from the correlation functions.

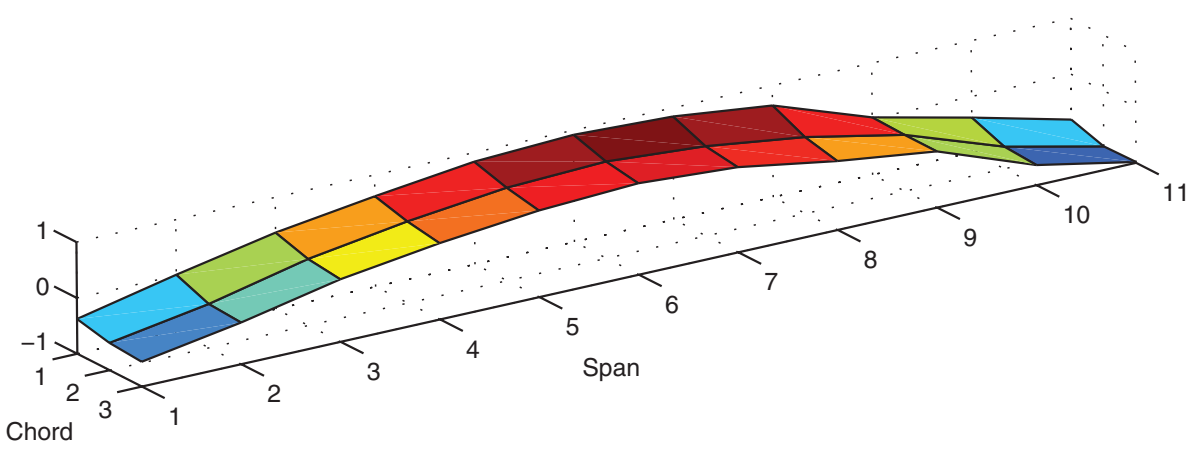

Figure 12 First mode shape estimate of AB-204 helicopter blade from the PSDs functions.

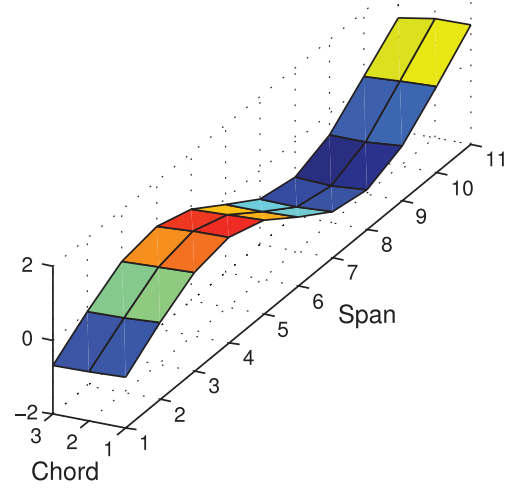

Figure 13 Second mode shape estimate of AB-204 helicopter blade from the PSDs functions.

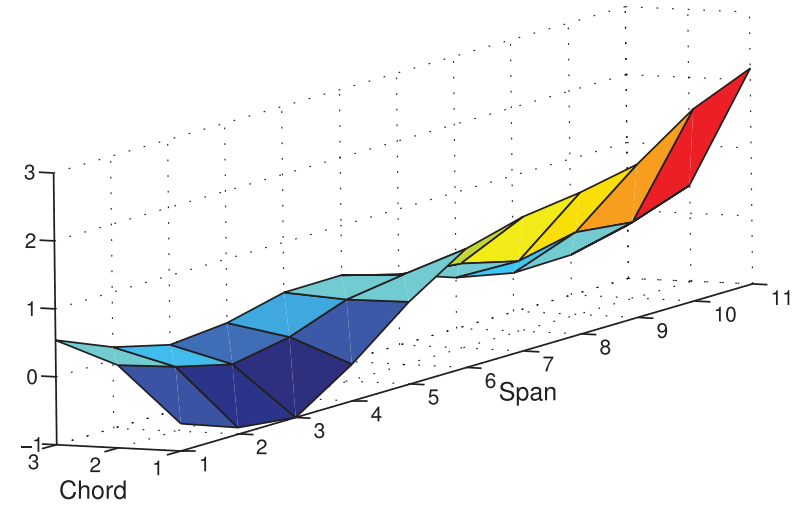

Figure 14 Fourth mode shape estimate of AB-204 helicopter blade from the PSDs functions.

Experimental Techniques (2013) @ 2013, Society for Experimental Mechanics 
frequency band of interest-must be valid, at least for each session of measurements. In fact, as shown for the helicopter blade, when a point of measurement is fixed, all the data can be reduced in order to get the modes (only four accelerometers were used along with the rowing technique). The data achieved by fitting, in the frequency domain, the PSDs pointed out as the bias due to the triangular window present in the correlations of finite time sequences could provide higher evaluations of the damping factors, because the spectra consider all the triangular window. On the contrary, the estimation in the time domain, limited at the first part of the correlation functions, could limit this bias effect. It is obvious that much lower decay rates, for a given length of the time sequences, bring to much higher errors in the damping ratio evaluations. In any case, to minimize the effect of the triangular window it is necessary to consider only the first parts of the correlation functions. So, a high number of shorter sequences permits to eliminate the residual random noise, on the other hand a low number of longer time functions allows one to minimize the bias effect due to the triangular window (with the same decay rates of the considered modes). In conclusion, the approach in the time domain, that directly uses the correlation functions, seems to be straighter and more efficient in the estimation of modal parameters, for systems in their actual working conditions, with respect to other methods based on the analysis carried out on the PSDs.

\section{Acknowledgments}

This work has been partially granted by the University of Rome "La Sapienza", Scuola di Ingegneria Aerospaziale Faculty research "Stima dei parametri modali dalle sole risposte dinamiche di sistemi aerospaziali", and by the Ateneo Federato della Scienza e della Tecnologia research program "Sviluppo di metodologie Output-Only per l'identificazione di parametri dinamici in strutture elicotteristiche".

\section{References}

1. Hermans, L., and Van der Auweraer, H., "Industrial Applicability of Modal Analysis on Operating Data," Proceedings of NATO Advance Study Institute on Modal Analysis and Testing, Seimbra (P); May 3-15, 1998.
2. Brincker, R., Zhang, L., and Andersen, P., Modal Identification from Ambient Responses Using Frequency Domain Decomposition, XVIII IMAC, S. Antonio, TX, 2000.

3. Agneni, A., Balis Crema, L., and Coppotelli, G., "Time and Frequency Domain Model Parameter Estimation by Output Only Functions," Proceedings of International Forum on Aeroelasticity and Structural Dynamics, Amsterdam, NL, 2003.

4. Agneni, A., Balis Crema, L., and Coppotelli, G., "Output-Only Analysis of Structures with Closely Spaced Modes," Mechanical Systems and Signal Processing. 24(5): 1240-1249. Special Issue: Operational Modal Analysis (2010).

5. Agneni, A., Brincker, R., and Coppotelli, G., “On Modal Parameter Estimates from Ambient Vibration Tests," Proceedings of International Seminar on Modal Analysis-ISMA 2004, Leuven, 2004.

6. Agneni, A., Balis Crema, L., and Coppotelli, G., "Output-Only Analysis of Coupled-Mode Structures," Proceedings of the 1st International Operational Modal Analysis Conference, Copenhagen, DK, pp. 201-212; April 26-27 (2005).

7. Agneni, A., Balis Crema, L., and Coppotelli, G., "Use of Correlation Techniques in Output-Only Analysis," Proceedings of International Operational Modal Analysis Workshop, Portonovo (Ancona), Italy (2009).

8. Van Overschee, P., and De Moor, B., Subspace Identification for Linear Systems: Theory, Implementation, Applications, Kluwer Academic Publishers, Leuven, Belgium, (1996).

9. Peeters, B., and De Roeck, G., “Reference-Based Stochastic Subspace Identification for Output-Only Modal Analysis," Mechanical Systems and Signal Processing 13(6): 395-409 (1999).

10. Stearn, S.D., Digital Signal Analysis, Hayden Book Co., Rochelle Park, pp. 233-236 (1975).

11. Marple, S.L., Jr., Digital Spectral Analysis with Applications, Prentice Hall Inc., Englewoods Cliffs, pp. 146-149 (1987).

12. Kunt, M., Traitement numérique des signaux, Dunod, Lousanne, pp. 251-257 (1981).

13. Agneni, A., and Balis Crema, L., "Damping Ratio Estimates from Autocorrelation Functions," in Proceedings of Damping'91, San Diego, CA, pp. GCD1-GCD16, February 13-15 (1991).

14. Coppotelli, G., "Experimental Identification of the Structural Properties of an AB 204 Helicopter Blade and Finite Element Model Validation," Experimental Techniques 33(5): 25-34 (2009). DOI: 10.1111/j.1747-1567.2008.00447.x. 